\title{
Neurobiologische Grundlagen für die Beratung
}

\author{
Heidi Möller
}

Online publiziert: 8. Juli 2019

(C) Springer Fachmedien Wiesbaden GmbH, ein Teil von Springer Nature 2019

Die Neurowissenschaften boomen und halten folgerichtig Einzug in neuere Beratungskonzepte. Es stellt sich ganz radikal die Frage, ob Veränderungen von Deutungs- und Handlungsmustern, die wir im Coaching, in der Supervision und Organisationsberatung anstreben, ohne die Berücksichtigung der unterschiedlichen Ebenen des limbischen Systems, der Bindungstheorie, der somatischen Marker, Embodimenttheorie u.v.m. überhaupt denkbar sind. Kann wissenschaftlich fundierte Beratung ohne Wissen um Neuroplastizität, die Funktion des Stressverarbeitungssystems, des Selbstberuhigungssystems, des Impulshemmungssystems, des Motivationssystems und des Realitäts- und Risikowahrnehmungssystems auskommen? Oder handelt es sich z.B. beim „Neurocoaching“ schlicht um einen weiteren Hype, ein überschätztes Konzept, das bald schon wieder verschwunden sein wird?

Coaches müssen sich schnell ein Bild über die Persönlichkeit, die Affektwelt, die Arten des Denkens und Handelns, der Belastungen und Ressourcen der Coachingklient/innen machen, um maßgeschneiderte Interventionen anbieten zu können. Das Wissen darum, welche Konzepte und Methoden nachweislich wirken und aus neurowissenschaftlicher Sicht bei welchen Coachinganliegen Sinn machen, beschreibt Gerhard Roth in seinem Beitrag Integratives Coaching auf neurowissenschaftlicher Grundlage.

Der Leib als vernachlässigte Dimension in Beratungsprozessen wird von Alica Ryba ins Zentrum ihrer Ausführungen gestellt. Unbewusste und vorbewusst-intuitive Prozesse lassen sich qua rationaler Selbstreflexion wenig erreichen und verändern. Ihr Plädoyer: Die Coaching-Community setzt zu stark auf kognitive Zugänge und kann sich durch Leibinterventionen anreichern und dadurch effektiver werden. An-

Prof. Dr. H. Möller ( $\bowtie)$

Institut für Psychologie, Universität Kassel, Holländische Straße 36-38, 34127 Kassel, Deutschland

E-Mail: heidi.moeller@uni-kassel.de 
hand eines Blickes in die Geschichte der Körpertherapien zeigt die Autorin die Spielräume auf, die Coaches sinnvoll nutzen können.

Der praxisorientierte Beitrag von Annette Diedrichs beschreibt die Arbeit mit dem Züricher Ressourcenmodell (ZRM) in der Teamentwicklung. Das ZRM ist aus neurowissenschaftlicher Sicht geeignet, die unbewusste Dimension menschlichen Erlebens und Verhaltens zu erreichen. Durch das Finden von Motto-Zielen kann in Teamentwicklungsprozessen die Selbststeuerung des Systems verbessert werden. Vor allem kann bei Change-Maßnahmen durch diese Methodik an einer gemeinsam getragenen Haltung gearbeitet werden und die gemeinsame Ausrichtung der Teamaktivitäten erfolgen.

Torsten Voigt kontextualisiert unseren Schwerpunkt mit einer soziologischen Perspektive auf die Neurowissenschaften. Er stellt die Errungenschaften dieser transdisziplinär aufgestellten Forschungstradition dar, skizziert jedoch auch die Grenzen der Erkenntnismöglichkeiten. Er erläutert den Erfolg des neurowissenschaftlichen Zugangs in zahlreichen Feldern und bringt ihn in den Zusammenhang mit einem gesellschaftlichen Klima der Selbstoptimierung. Differenziert arbeitet er die Bedeutung der Neurowissenschaften für die Beratungswissenschaft auf, ohne nicht auch das Scharlatanerie-Problem fundiert zu beschreiben.

In diesem Heft tut sich ein zweiter kleinerer Themenschwerpunkt auf: Diversity in Fragen des Geschlechts, des Alters und der Kultur sind Querschnittsthemen jedweden Beratungsformats. Wie kann es gelingen, Unterschiede konstruktiv zu nutzen? Dieser Frage gehen Alexander Tirpitz, Angélique Zessin und Julia Hapkemeyer nach. Sie spannen einen Bogen zwischen Diversity als Managementaufgabe versus Diversity als Organisationsentwicklungsprozess. Hintergründe von Schwierigkeiten, Vielfalt zu nutzen, werden genauso aufgezeigt wie dessen Chancen. Konkrete Handlungsfelder für konstruktive Entwicklungsprozesse sind detailreich aufgeführt.

Laura E. Creon und Carsten C. Schermuly legen eine Klassifikation unterschiedlicher Diversity-Trainings vor. Das nahezu unüberschaubare Angebot unterschiedlicher Interventionen wird damit durchsichtiger, und vor allem gelingt es den beiden Autoren, die empirische Evidenz systematisch aufzuarbeiten. Der Beitrag stellt eine große Hilfe für Menschen mit Personalverantwortung, aber auch für Berater/innen dar, einen Weg durch den Dschungel der Möglichkeiten, Diversity als Ressource zu nutzen, zu finden.

In einem Praxisbericht beschreiben Dorette Eichler und Sibylle Rothkegel ein außergewöhnliches Supervisionsprojekt in einem der belastetsten Arbeitsfelder, die zumindest ich mir vorstellen kann. Das Auswärtige Amt als Arbeitgeber unterstützt die Mitarbeitenden in den Auslandsvertretungen, die die Visaanträge syrischer und jesidischer Flüchtlinge bearbeiten, durch Supervisionsangebote. Der Erfahrungsbericht bringt uns ein Arbeitsfeld nahe, das gekennzeichnet ist von schwierigster Beziehungsgestaltung, psychischen Belastungen und ethischen Dilemmata. Mit einem psychodynamischen Konzept konnte es gelingen, diese Herausforderungen besser zu bewältigen.

Der Themenschwerpunkt Digitalisierung im Heft 3/18 hat mehrere Autor/innen zu Ergänzungen und kritischen Reflexionen angeregt. So haben Henrike Barth und Thomas Bachmann in einer schriftlichen Befragung das Nutzungsverhalten und die Nutzungsintention von Coaches untersucht. Es wird die Frage beantwortet, welche 
digitalen Angebote die Coaches machen, welche Einstellung sie zu diesen Medien haben und wie gesellschaftliche, organisationale und subjektive Normen das Beraterverhalten an dieser Stelle beeinflussen. Dabei nutzten sie die bewährte Theorie des geplanten Verhaltens (Ajzen), um die Interdependenzen zu klären. Diese höchst ambivalent besetzte Herausforderung des Beratungsmarktes wird uns in der OSC immer wieder beschäftigen, da die Digitalisierungsfrage mit heißen Emotionen in der Beratungsszene diskutiert wird.

Im Diskurs antwortet Ursula Wagner auf den Beitrag von Beate Fietze und Thomas Bachmann zur Digitalisierung im Coaching und nimmt dabei eine ermunternde Perspektive ein. Sie ermutigt die Coaching-Community, sich mit Online-Formaten anzufreunden. Vor allem stellt sie die Möglichkeiten heraus, die der Einsatz neuer Medien innerhalb und außerhalb der Organisationen hervorbringt. Sie sieht es als unser aller Herausforderung, mit intelligenten Blended-Angeboten Innovation in die Beratungsbranche zu bringen. 